Title:

\title{
A Mesenchymal Tumor Cell State Confers Increased Dependency on the BCL-XL Anti-apoptotic Protein in Kidney Cancer
}

Authors: Treg Grubb ${ }^{1,2, \uparrow}$, Smruthi Maganti ${ }^{1,2, \uparrow}$, John Michael Krill-Burger ${ }^{3}$, Cameron Fraser $^{4,5}$, Laura Stransky ${ }^{6}$, Tomas Radivoyevitch ${ }^{7}$, Kristopher A. Sarosiek $^{4,5}$, Francisca Vazquez $^{3}$, William G. Kaelin, Jr. ${ }^{6,8, *}$, and Abhishek A. Chakraborty ${ }^{1,2, *}$

\section{Affiliations:}

${ }^{1}$ Department of Cancer Biology, Lerner Research Institute, Cleveland Clinic, Cleveland, $\mathrm{OH} 44195$, USA.

${ }^{2}$ Case Comprehensive Cancer Center, Case Western Reserve University, Cleveland, $\mathrm{OH} 44106$, USA.

${ }^{3}$ Broad Institute of Harvard and MIT, Cambridge, MA 02142, USA.

4John B. Little Center for Radiation Sciences, Harvard T.H. Chan School of Public Health, Boston, MA 02115, USA.

${ }^{5}$ Department of Environmental Health, Harvard T.H. Chan School of Public Health, Boston, MA 02115, USA.

${ }^{6}$ Department of Medical Oncology, Dana-Farber Cancer Institute and Brigham and Women's Hospital, Harvard Medical School, Boston, MA 02215, USA.

${ }^{7}$ Quantitative Health Sciences, Lerner Research Institute, Cleveland Clinic, Cleveland, $\mathrm{OH} 44195$, USA. 
${ }^{8}$ Howard Hughes Medical Institute, Chevy Chase, MD 20815, USA.

${ }^{\top}$ These authors contributed equally.

*Correspondence should be addressed to W.G.K (william_kaelin@dfci.harvard.edu) and A.A.C (chakraa@ccf.org)

One Sentence Summary: Cell state, but not pVHL and/or HIF status, defines the dependency of kidney cancer cells on the BCL-X $\mathrm{L}$ anti-apoptotic protein. 


\section{Abstract}

Genome-wide genetic screens have identified cellular dependencies in many cancers. Using the Broad Institute's Achilles shRNA screening dataset, we mined for targetable dependencies by cell lineage. Our studies identified a strong dependency on BCL2L1, which encodes the BCL-X $X_{L}$ anti-apoptotic protein, in a subset of kidney cancer cells. Genetic and pharmacological inactivation of BCL-X $\mathrm{L}$, but not the related anti-apoptotic proteins BCL-2, led to fitness defects in renal cancer cells, and also sensitized them to chemotherapeutics. Neither BCL-XL levels (absolute or normalized to BCL-2) nor the status of the $V H L$ gene, which is frequently mutated in kidney cancer, predicted BCL-XL dependence. Transcriptional profiling, however, identified a 'BCL- $\mathrm{K} L$ dependency' mRNA signature, which included elevated mesenchymal gene expression in BCL-XL dependent cells. Promoting mesenchymal transition increased BCL-X $\mathrm{L}$ dependence; whereas, conversion to a more differentiated state overcame $B C L-X L$ dependence in kidney cancer cells. The 'BCL-XL dependency' mRNA signature was observed in almost a third of human clear cell Renal Cell Carcinomas (ccRCCs), which were also associated with worse clinical outcomes. Finally, an orally bioavailable $B C L-X_{L}$ inhibitor, A-1331852, showed anti-tumor efficacy in vivo. Altogether, our studies uncovered an unexpected link between cancer cell state and dependence on the anti-apoptotic BCL$\mathrm{X} L$ protein and justify further testing on $B C L-X\llcorner$ blockade as a potential way to target a clinically aggressive subset of human kidney cancers. 


\section{INTRODUCTION}

Renal Cell Carcinoma (RCC) ranks among the top ten forms of cancer in humans, with current estimates projecting $\sim 79,000$ new diagnoses and $\sim 14,000$ deaths this year in USA (1). Early stage RCC, which is observed in nearly $60 \%$ of patients, can be effectively managed with either surgery or surveillance; however, nearly 4 in 10 patients present with advanced or metastatic RCC, and experience much poorer outcomes. Moreover, almost a third of the patients with higher-risk localized disease during initial diagnosis subsequently develop metastatic disease. Therapeutic options to manage advanced disease presently include combining a checkpoint inhibitor (CKI) either with another CKI or with a tyrosine kinase inhibitor (TKI), and the most recent trials have shown that between $30-60 \%$ patients exhibit measureable responses with these therapeutic combinations (2-4). However, there is limited recourse for the remaining patients. Finally, most patients eventually develop resistance to these treatments and subsequent treatment options are largely ineffective.

The clear cell Renal Cell Carcinomas (ccRCCs) represent $\sim 70-75 \%$ of RCCs (5). Inactivation of the von Hippel-Lindau tumor suppressor protein ( $\mathrm{pVHL}$ ) is a nearly universal hallmark of ccRCC (5), and mechanistic studies defining the consequences of pVHL loss have informed the pre-clinical discovery of actionable dependencies in ccRCC (6-8). pVHL functions as the substrate recognition subunit in an E3 ligase complex that is perhaps best known to mediate oxygen-dependent destruction of the $\alpha$ subunit of the Hypoxia Inducible Factor (HIFa) $(9,10)$. Consequently, HIFa accumulates in pVHL-deficient ccRCCs, independent of oxygen availability. There are two transactivation-competent isoforms of HIF $(\mathrm{HIF} 1 \alpha$ and HIF2 $\alpha)$ and chronic activation of 
HIF2 $\alpha$ drives kidney cancer pathogenesis $(11,12)$. Therapeutic strategies to target HIF2 $\alpha$ directly have been developed in the last decade $(13,14)$. These approaches have shown promise in both pre-clinical $(15,16)$ and clinical settings $(17,18)$. But not all patients respond, and not all responses are durable. Therefore, new treatments, especially to target tumors that are refractory to HIF2 $\alpha$ inhibitors (and other therapies), are urgently needed.

Recent largescale screening efforts, such as the Broad Institute's Achilles (19) and DepMap projects (20), and Novartis' DRIVE project (21), have provided investigators with publically accessible resources that enable the identification of novel oncogenic dependencies. These screening strategies rely on either genetic (e.g. shRNA and CRISPR/Cas9) or pharmacological tools. These different tools can produce different phenotypes, even when directed at the same target. For example, CRISPR/Cas9-based screens routinely yield phenotypes associated with near complete elimination (knockout) of the gene product. In contrast, shRNA screens often score phenotypes associated with partial mRNA reduction (knockdown), and perhaps better model the incomplete blockade of target proteins that is typically achieved with drugs. Importantly, though, the candidates identified through shRNA-based approaches need to be rigorously vetted because of the notorious "off-target" cytotoxicity associated with shRNA use (22).

Here, we exploited the availability of multiple genetic screening databases to identify novel targetable dependencies in kidney cancer. We reasoned that the genetic lesions acquired during renal oncogenesis and/or the master regulators of lineage specification could confer targetable dependencies in these cancer cells. Therefore, a 
comparison of genetic dependencies in the kidney lineage cells could identify targetable vulnerabilities that are preferentially enriched in RCC (versus other lineages).

\section{Results}

$B C L-X_{L}$ is a lineage-specific dependency in kidney cancer

To identify dependencies that are selectively enriched in kidney cancer cells (versus all other lineages), we used the Broad Institute's (shRNA-based) Achilles dataset. This dataset measures the enrichment or depletion of shRNAs in a large representative sample set of cancer cells representing various cancer types and lineages. The cell lines in this dataset were lentivirally transduced in a pooled format with a genome-wide shRNA library and cultured over 6-8 weeks to track changes in the abundance of shRNAs by next-gen sequencing (19).

Given the concern of losing critical dependencies because they failed to score in all of the cell lines due to inherent genetic heterogeneity (i.e. false negatives), we sorted dependencies that showed strong selectivity in at least a subset of kidney lineage cells [Likelihood Ratio Test (LRT)>100]. We then excluded "common essential genes", which typically have housekeeping roles, and confirmed that the scored dependency was observed in a minimum of three independent cell lines within the kidney lineage (Table S1), thereby scoring twenty potential dependencies. We then ranked the dependencies by LRT and interrogated the top ten "hits" from this analysis (Fig. 1A). We noted that the top two candidates were $H N F 1 B$ and $P A X 8$, both of which have an established role in kidney lineage specification $(23,24)$. Most of the other candidates on 
the list included ribosomal subunits, which are not inherently druggable; however, $B C L 2 L 1$, scored as the top gene that encoded a druggable gene product. The impact of BCL2L1 loss by shRNA on kidney cancer cells was confirmed using the more recent CRISPR/Cas9 dependency maps (20). We observed a great degree of similarity in BCL2L1 dependence between the two datasets and confirmed that a subset of kidney cancer cells (e.g. CAKI-2, TUHR4TKB, and TUHR10TKB) showed very strong dependence on BCL2L1 in both datasets (Fig. 1B).

The BCL2L1 gene encodes the anti-apoptotic BCL- $\mathrm{X}_{\mathrm{L}}$ protein, a member of the broader BCL-2-family of proteins, which includes BCL-2, BCL-w, MCL-1, and BFL-1/A1. To confirm our initial observations, we lentivirally transduced representative ccRCC cell lines (A-498, CAKI-2, UMRC-2, and UMRC-6) with three unique shRNAs that targeted either BCL2L1 (499, 500, and 503), BCL2 (069, 303, and 675), or non-targeting controls (shCon). After selection in puromycin, we stained the surviving cellular population with crystal violet. Knockdown of BCL2L1 caused evident cytotoxicity in A-498, CAKI-2, and UMRC-6 within 3-5 days post selection and in UMRC-2 cells $\sim 10-12$ days post selection (Fig. 1, C and D). In contrast, BCL2 shRNAs did not cause such profound cytotoxicity in the kidney cells, despite robustly knocking down BCL2 (Fig. 1, C and D).

Two BCL-XL targeting shRNAs (499 and 500) also partially downregulated BCL2. To formally establish that the cytotoxic effects of these $B C L 2 L 1$ shRNAs were "ontarget", we first engineered a BCL-XL CDNA that harbored silent mutations in the sh499 recognition sequence. Expression of this construct (499R) rescued the cytotoxicity associated with sh499, but not sh500, in two different cell lines (Fig. 1, E to G). We then introduced additional silent mutations into the 499R backbone to also make this cDNA 
resistant to sh500 (499/500R). Expression of this BCL-XL CDNA rescued the cytotoxicity associated with both sh499 and sh500 in both the renal cell lines (Fig. 1, E to G). Together, these genetic studies confirmed that ccRCCs are dependent on the BCL-XL anti-apoptotic protein, but less so on the closely related BCL-2 protein; and indicated that the inherent sensitivity of ccRCCs might differ between cell lines, with cells like UMRC-2 presenting with delayed cytotoxicity in response to BCL-XL loss.

Pharmacological BCL-XL blockade mimics results of genetic studies

The BCL-2-family anti-apoptotic proteins have been targets of clinical interest for many years and, as such, have been subjected to intensive "hit-to-lead" campaigns to identify highly specific pharmacological inhibitors. These efforts have identified the BCLXL/BCL-2-dual inhibitors ABT-737 (25) and ABT-263 (Navitoclax) (26), the BCL-2specific inhibitor ABT-199 (Venetoclax) (27), and more recently the BCL-XL-specific inhibitor A-1331852 (28).

To validate the findings from our genetic studies, we characterized a panel of ten ccRCC lines for sensitivity to the BCL-2-family inhibitors. We observed that cell lines that were exquisitely sensitive to BCL-XL loss in genetic studies (e.g. CAKI-2 and TUHR4TKB) were also highly sensitive to three days of treatment with either ABT-263 (fig. S1) or the BCL-XL-specific inhibitor A-1331852, with cellular $\mathrm{IC}_{50}$ values in the low $\mathrm{nM}$ range (Fig. 2A and fig. S2A). In contrast, cell lines like UMRC-2, which showed delayed response to genetic BCL-X $\mathrm{L}$ loss, were likewise less responsive to three days of treatment with pharmacological agents that target BCL- $\mathrm{X}$ (Fig. $2 \mathrm{~A}$ and figs. $\mathrm{S} 1$ and S2A). These analyses also identified the A-498 and SLR23 cells as additional examples 
of $A-1331852$ sensitive ccRCCs. The BCL-XL dependency in these cells was specific; because, in contrast to the BCL-2-dependent B-cell lymphoma line SU-DHL-6, the ccRCC lines were virtually resistant to the BCL-2 inhibitor ABT-199 (Fig. 2B and fig. S2B). Altogether, nearly $40 \%$ of the $c c R C C$ lines were sensitive $\left(\mathrm{IC}_{50}\right.$ in the $\mathrm{nM}$ range) to A-1331852. Importantly, sensitivity in only a subset of cell lines is a common occurrence, perhaps due to underlying genetic heterogeneity among cell lines. For instance, the HIF2 $\alpha$ inhibitor, which has recently received FDA approval for use against ccRCCs, efficiently targets only a subset of $\sim 20-30 \%$ ccRCCs.

We confirmed that the cytotoxicity triggered by A-1331852 treatment occurred because of increased apoptosis. Upon A-1331852 treatment, Annexin V (AnnV) versus Propidium lodide staining showed a significant increase in apoptotic population in the BCL-XL-inhibitor sensitive CAKI-2 and A-498 cells, but not the relatively insensitive UMRC-6 and UMRC-2 cells (fig. S3). Together, these results demonstrated that pharmacological tools produced results that were largely consistent with our genetic studies and linked the dependency of ccRCCs on BCL-XL's anti-apoptotic role.

We next attempted to validate these findings using an orthogonal assay. Antiapoptotic BCL-2 family proteins exert their function by binding and sequestering proapoptotic "BH3-only" proteins (e.g. BIM, BID, and PUMA) or the pore-forming proteins (e.g. BAX and BAK). Synthetic, pro-apoptotic $\mathrm{BH} 3$ peptides can mimic or block these interactions and trigger cellular apoptotic programs. Probing for hallmarks of apoptosis, such as mitochondrial membrane depolarization that is indicative of cytochrome $\mathrm{c}$ release, in response to these peptide treatments can measure cellular dependencies on specific anti-apoptotic BCL-2-family proteins ex vivo (29). Using this "BH3 profiling" 
approach, which monitors mitochondrial potential using the JC1 dye, we compared the dependencies of ccRCCs on BCL-2-family anti-apoptotic proteins. In line with our pharmacological experiments, we observed that the $\mathrm{BCL}-\mathrm{X} \mathrm{L}$ dependent $\mathrm{CAKI}-2$ cells showed significant membrane depolarization with agents that blocked BCL-XL's interaction with downstream effectors (e.g. the Hrk peptide and the Wehi539 pharmacological inhibitor), but not with MCL-1 blockers (MS1 peptide) (Fig. 2C). In contrast, the UMRC-2 cells, which were less responsive to acute BCL-XL inhibition, were also largely non-responsive to agents that disrupt BCL- $\mathrm{XL}_{\mathrm{L}}$ function (Fig. 2D). These results demonstrated that $B C L-X_{L}$ actively restrains pro-apoptotic signaling in ccRCCs, leading to a dependence on this protein for continued survival.

$B C L-X_{L}$ inhibition sensitizes kidney cells to chemotherapeutic agents

RCCs have historically been recognized for their resistance to traditional chemotherapeutics (30), most of which function by promoting apoptosis. We hypothesized that BCL-X $\mathrm{L}$ function, which has been previously shown to be a drug resistance mechanism in many cancers, could be a physiological barrier to chemotherapeutic response in ccRCC. To address this hypothesis, we treated the A1331852-insensitive UMRC-2 and OSRC2 cells with various dosage combinations of A1331852 and chemotherapeutics such as 5-FluoroUracil (5-FU), Docetaxel, and Doxorubicin. Many statistical models exist to analyze drug-drug interaction matrices to score for potential synergy or antagonism (e.g. HSA, Loews, Bliss, and ZIP) (31-34). To overcome the caveats associated with these individual models, open source R-based packages have been generated, which simultaneously compare the dataset across 
multiple models. We used a recently developed we application that provides visualization of the SynergyFinder package (35). This analysis demonstrated synergy at many dose combinations between the $B C L-X_{L}$ inhibitor and all three chemotherapy drugs using all the models, which we then aggregated to identify doses of potential synergy between A-1331852 and the corresponding chemotherapeutic agent (Fig. 3, fig. S4, and Table S2). Together, our drug-drug interaction studies showed that BCL-XL inhibition chemosensitized ccRCCs to a wide range of chemotherapeutic agents.

\section{Epithelial-Mesenchymal Transition (EMT) is Associated with Bcl-xL Dependency}

Our findings suggested that a subset of $\sim 40 \%$ ccRCC lines were sensitive to acute $B C L-X_{L}$ inhibition. Therefore, we interrogated the biomarkers that would allow differentiating cCRCCs based on BCL- $X_{L}$ dependence. To this end, we first mined the Broad Achilles dataset and correlated mRNA levels of BCL2L1 in different lineages to their respective $B C L-X_{L}$ dependency using Pearson's correlation coefficient. This analysis showed a weak correlation $\left(r^{2}<0.3\right)$ between $B C L 2 L 1$ mRNA expression levels and BCL-XL dependency (fig. S5A). Next, we compared the protein expression levels of $B C L-X_{L}$ and its closely related family member, $B C L-2$, in our panel of $C C R C C$ lines. Consistent, with the mRNA correlation analysis, we did not find any significant difference in BCL-XL protein expression levels among the A-1331852 sensitive versus insensitive ccRCC lines (fig. S5B). We then compared the relative abundance of BCL-2 and $B C L-X_{L}$ and observed that $B C L-X_{L}: B C L-2$ ratio was also not a predictor of $B C L-X_{L}$ dependency in ccRCC (fig. S5, C to E). 
To identify determinants of BCL-XL dependency in an unbiased manner, we performed transcriptomics studies. We chose two representative sensitive lines (A-498 and CAKI-2) and two insensitive lines (OSRC-2 and UMRC-2). We transcriptionally profiled, using RNA-Seq, these ccRCC cells under (untreated) native growth conditions and also after acute treatment with A-1331852 (Fig. 4A). We reasoned that this approach would not only allow us to measure innate differences among cells that might underlie BCL-XL dependence, but also potential differences in cellular response to acute treatment with BCL-XL inhibitors. For these experiments, we empirically determined A1331852 treatment time-points (typically 6-16 hour treatments) that preceded any overt cytotoxicity, which we feared could confound our gene expression studies.

We analyzed the transcriptional signatures using the EdgeR module in $\mathrm{R}$ and identified genes that were differentially expressed among the sensitive (S) versus insensitive (I) lines in both the untreated and the A-1331852-treated condition (Fig. 4A and Tables, S3 and S4). Among these differentially expressed genes, we compared enrichment or depletion of annotated gene sets described in mSigDB (36), using Gene Set Enrichment Analysis (37). These studies found that, compared to the insensitive cells, prominent differences existed innately in untreated sensitive cells (Fig. 4B and Table. S5); however, the extent of many these differences were further amplified upon BCL-X inhibition (Fig. 4B and Table. S6). These gene sets represented a number of biological pathways, including p53 response, cytokine response (e.g. interferon response), and cell state pathways [e.g. epithelial to mesenchymal transition (EMT)] (Fig. 4, B to D, and Tables, S5 and S6). 
When activated by cellular damage or stress, p53 transcribes multiple proapoptotic proteins to trigger cell death in cells that are primed for apoptosis $(38,39)$, and favors cell death induction by inhibitors of $B C L-X_{L}(40)$. We therefore reasoned that intact p53 signaling may be associated with BCL-X dependence and potentially act as a predictor of $\mathrm{A}-1331852$ response. We thus tested whether the segregation of $\mathrm{ccRCC}$ lines either into BCL-XL-dependent or independent subsets was p53-dependent. First, we mined the Broad Institute's Cancer Cell Line Encyclopedia (CCLE) to evaluate p53 mutation status in our panel of ccRCC lines. This analysis showed that 3 of the 4 sensitive cell lines had wild-type p53; however, the p53-mutant SLR23 cells were also highly sensitive to BCL-XL loss (fig. S6A). In contrast, cell lines that were insensitive to BCL-X inhibition included OSRC2 and UOK101 cells, both of which have wild-type p53 (fig. S6A). Therefore, p53 status alone appeared to be insufficient to predict BCL-XL dependency.

To probe this further, we treated ccRCC lines with doxorubicin and measured the accumulation of total p53, phosphorylated p53, and the p53-target gene CDKN1A, which encodes the cyclin-dependent kinase inhibitor p21. We found that the basal levels of $\mathrm{p} 21$, and its induction in response to doxorubicin, was lower in many of the intermediate and insensitive ccRCC lines, compared with the sensitive cells (fig. S6B). UOK101 cells were, once again, a notable exception with significant p21 expression but little BCL-XL dependence, perhaps due to other mutations that block apoptosis in this cell line. Altogether, we concluded that p53 response to DNA damaging agents could partly predict BCL-XL dependency in CcRCC lines. 
Finally, we focused our attention on the cell state pathways because of the relative novelty of epithelial-mesenchymal transition (EMT) as a determinant of BCL-XL dependency. To validate our RNA-Seq data, we used flow cytometry to compare the expression levels of CD44, a well characterized marker of mesenchymal state, in ccRCC cells that were either sensitive or insensitive to acute $B C L-X_{L}$ inhibition. Consistent with our transcriptional studies, we observed that CD44 expression levels were significantly elevated in BCL-XL dependent cells (fig. S7A). These studies suggested that mesenchymal tumor cell state was indeed a promising biomarker of BCL-XL dependence.

We then addressed the functional causality of this pathway in driving apoptosis upon A-1331852 treatment. We reasoned that a causal role for cell state differences in conferring $B C L-X_{L}$ dependence could be established if downregulating mesenchymal features in sensitive cells promoted insensitivity to A-1331852; or conversely, if inducing mesenchymal features in insensitive lines was sufficient to sensitize them to A-1331852 treatment. To this end, we first treated the sensitive lines, A-498 and CAKI-2, with alltrans-Retinoic Acid (ATRA) for three days with the hope that the differentiation promoting effects of ATRA would diminish mesenchymal features in these cells (Fig. 5A). Indeed, we noted a small, but statistically significant, reduction in CD44 expression in both cell lines (Fig. 5B). Interestingly, this minimal change in mesenchymal was still sufficient to reduce the sensitivity of both $A-498$ and CAKI-2 cells to A-1331852 treatment (Fig. 5C).

Next, we designed an experiment to address mesenchymal sufficiency. We began by treating the A-1331852-insensitive, UMRC-2 and OSRC-2, cells for 3 days 
with TGF $\beta$, a potent inducer of mesenchymal transition (Fig. 5D). Unfortunately, we did not find any changes in CD44 expression in OSRC2 cells and thus substituted these cells with the intermediate-sensitive UMRC-6 cells. In both UMRC-2 and UMRC-6 cells, TGF $\beta$ treatment led to robust induction of CD44 (Fig. 5E). Strikingly, promoting mesenchymal features also increased A-1331852 sensitivity in both these lines (Fig. 5F). Importantly, these results were specific to $B C L-X_{L}$ dependence because perturbation of cell state failed to confer sensitivity to pharmacological BCL-2 inhibition (fig. S7, B and C). Altogether, these studies demonstrated that a mesenchymal signature did not merely correlate with increased BCL-XL dependency but was both necessary and sufficient to modulate the response of CCRCC cells to BCL-XL blockers.

Previous literature suggested that chronic HIF activation in pVHL-deficient ccRCCs status could impact apoptotic response. Many transcriptional targets of HIF are known regulators of cell death pathways, including $B C L 2 L 1$ in certain cellular contexts $(41,42)$. Moreover, in certain cell lines, pVHL expression has been shown to impact EMT $(43,44)$. Therefore, we addressed if $\mathrm{pVHL}$ status could influence BCL-XL dependency. We lentivirally transduced pVHL-deficient ccRCC cells to restore expression of wild-type pVHL (VHL) or an empty vector control (VEC), and thus generated isogenic CAKI-2 and A-498 cells that either did or did not express pVHL (Fig. S8A). Reintroduction of $\mathrm{pVHL}$ does not lead to fitness defects in vitro under standard cell culture conditions, but impedes ccRCC tumor growth in vivo, enabling in vitro studies that address the influence of $\mathrm{pVHL}$ status. Surprisingly, reintroduction of $\mathrm{pVHL}$ in CAKI2 and A498 cells did not alter their mesenchymal cell state (fig. S8B), and consistent with these observations failed to impact the sensitivity of these cells to 
pharmacological BCL- $X_{L}$ inhibition (fig. S8, C and D). These results indicate that the impact of $\mathrm{pVHL}$ on EMT is likely dependent on biological context, and $\mathrm{pVHL}$ status is not sufficient to predict BCL-XL dependence in kidney cancer cells.

The EMT Signature Predicting BCL- $X_{L}$ Dependence is a Clinically-exploitable Feature

Based on the importance of the cell state pathways as determinants of $B C L-X_{L}$ dependency, we next probed for the prevalence of this signature in clinical samples. We generated a list of differentially-expressed genes (DEG) by comparing the relative expression patterns between the A-1331852-sensitive and insensitive ccRCC cell lines (Figs. 4A and 6A). We then analyzed kidney cancer gene expression data mined from The Cancer Genome Atlas (TCGA) and noted, as expected, that principal component analysis led to segregation of the RCC tumors into three distinct clusters, each representing one major RCC disease subtype (Fig. 6B and fig. S9). We then integrated the DEG list predicting $B C L-X L$ dependence with the $c c R C C$ tumor cluster. This analysis allowed us to establish (a) the prevalence of the BCL-XL dependency signature (Fig. $6 \mathrm{C})$, and (b) the differences in amplitude of the individual gene sets that together comprised the BCL-XL dependency signature, specifically in human clear cell renal tumors. We observed that the BCL-XL dependency signature (red) was observed in $\sim 30 \%$ of human ccRCCs. The EMT signature was among the most differentially expressed gene signatures among these cohorts; whereas, differences in the p53 pathway, albeit statistically significant, showed more subtle differences (Fig. 6D). Finally, as expected from the typically aggressive nature of mesenchymal tumors, the 
BCL-X dependency signature was associated with worse clinical outcomes (Fig. 6E).

These studies suggested that mRNA gene expression could be exploited as a biomarker to identify patient tumors exhibiting features of $B C L-X L$ dependence.

\section{$B C L-X_{L}$ Inhibition Impedes In Vivo Tumor Growth}

The BCL-XL-selective inhibitor, A-1331852, has been previously shown to be orally bioavailable in subcutaneous tumor xenografts (45). Therefore, A-1331852 treatment could be used to interrogate the therapeutic feasibility of BCL-XL inhibition in ccRCC. Unfortunately, several ccRCC lines, including the highly BCL-XL-dependent CAKI-2 and A-498 cells, have poor engraftment rates as subcutaneous tumors in $\mathrm{NCR}^{\text {nu/nu }}$ mice. The UMRC-2 cells, however, engraft tumors readily. Interestingly, we found that, as seen with our genetic studies, sustained pharmacological BCL-XL inhibition (>2 weeks) led to $\mathrm{nM} I \mathrm{CC}_{50}$ in UMRC-2 cells (fig. S10). These results justified the use of UMRC-2 cells for in vivo studies, which typically rely on dosing regimens extending to 3-4 weeks.

We inoculated UMRC-2 cells subcutaneously into 7 week old NCR ${ }^{\text {nu/nu }}$ mice (equal number of males and females) and allowed tumor engraftment and growth for $\sim 6-8$ weeks. Tumor-bearing animals $\left(>100 \mathrm{~mm}^{3}\right)$ were randomized to receive either 25 $\mathrm{mg} / \mathrm{kg}$ A-1331852 or sham-vehicle control, twice a day, orally, for up to 4 weeks. Tumor volumes were measured at the point of enrollment (Fig. 7A) and then weekly after dosing was initiated (Fig. 7B). We noted a significant decrease in tumor growth in A1331852 treated mice, as noted in smaller tumor volumes (Fig. 7, B to D). Histological 
analysis of the harvested tumors showed zones of necrotic cells in A-1331852 treated tumors. These necrotic regions were marked by elevated Cleaved Caspase 3 staining, consistent with apoptotic cell death triggered by A-1331852 treatment (Fig. 7, E and F).

The animals handled the dosing without any notable behavioral or body weight changes (fig. S11A). However, we noted profound splenomegaly in A-1331852 treated mice (Fig. 7D and fig. S11B). Histological analysis confirmed a notable disruption of splenic tissue architecture in A-1331852-treated animals, including a virtual absence of germinal centers (fig. S11, C and D). In contrast to the spleen, however, the histology and macroscopic appearance of other organs (e.g. kidney, liver, lung, and heart) were virtually indistinguishable between the vehicle and A-1331852 treated cohorts (fig. S12). Altogether, these findings were promising despite the technical necessity to employ the relatively insensitive UMRC-2 cells, and demonstrated the overall feasibility of BCL-XL inhibition as a strategy to block ccRCC tumor growth.

\section{DISCUSSION}

Despite recent advances in therapy, metastatic kidney cancer is ultimately incurable, and there is a recognized need for new therapeutic approaches to complement existing treatments. To address this need, in this study we interrogated lineage-specific dependencies. We made two modifications to a standard analytical pipeline. First, rather than probing for 'synthetic lethality' dependencies that are associated with a specific oncogenic alteration (e.g. truncal genetic lesion), we mined for candidate dependencies that are preferentially enriched in a given lineage, here in 
kidney cancer. Second, within the lineage, we sorted for - and retained - strongly selective dependencies even in a subset of cells. This strategy enabled the identification of candidate "hits" that were strong subset-specific dependencies in kidney cancer cells, including $B C L 2 L 1$, which encodes the anti-apoptotic BCL-XL protein.

We focused on BCL-XL because it was both druggable and already had many well characterized pharmacological inhibitors available, which could facilitate faster adaptation into the clinic. However, other genes in our list (e.g. ITGAV) also encode druggable proteins and warrant further study. Moreover, some otherwise "undruggable" proteins (e.g. HNF1B and PAX8) could be targeted with drugs that promote their proteolysis, such as deubiquitinase inhibitors and/or proteolysis-targeting chimeras (PROTACs). Notably, PROTACs have also been developed for BCL-XL, in an attempt to reduce drug resistance and to reprogram $B C L-X_{L}$ inhibition away from platelets, where it leads to undesirable cytotoxic side effects.

We validated $B C L-X \mathrm{~L}$ as a dependency in $\mathrm{CCRCC}$ multiple ways. First, we confirmed that ccRCC lines, such as CAKI-2 and TUHR4TKB, which were exceptionally sensitive to BCL2L1 in the Achilles dataset, were also independently identified as BCL$\mathrm{X}_{\mathrm{L}}$ dependent in the Novartis DRIVE genome-wide shRNA screen and the Broad Institute's CRISPR/Cas9 dependency map. Next, using three different shRNAs, we confirmed fitness defects in multiple ccRCC cell lines upon loss of BCL-XL, but not its closely related sibling BCL-2. Finally, we demonstrated that these genetic effects were "on-target" by rescuing the cytotoxicity associated with two of these shRNAs.

To develop upon our genetic studies, we employed pharmacological agents that target BCL-X. We found remarkable overlap in the sensitivity of cell lines using either 
one of these approaches. We also noted that some cell lines (e.g. UMRC-2) showed cytotoxicity only upon chronic BCL-XL blockade. Importantly, we did not observe any measurable response to the BCL-2 inhibitors, demonstrating the specificity of $B C L-X L$ dependence in ccRCC.

Kidney cancer, until recently, was curable only by surgical resection. This was largely because standard chemotherapeutic agents, including topoisomerase inhibitors, taxanes, and nucleoside analogues failed to show any significant clinical efficacy in many independent trials (30). The ability to promote apoptosis is central to the efficacy of many traditional chemotherapeutic agents. We therefore addressed if the antiapoptotic function of $B C L-X_{L}$ could be a physiological barrier to chemotherapy response, and thus underlie the observed insensitivity of ccRCCs to these agents. Consistent with this idea, our drug-drug interaction studies showed synergistic interactions between the $B C L-X_{L}$ inhibitor $A-1331852$ and a number of traditional chemotherapeutics. Therefore, BCL-XL inhibition sensitizes RCCs to chemotherapy, and offers us clinically usable opportunities to target RCCs that are non-responsive to other therapeutic strategies.

The importance of anti-apoptotic proteins has been previously interrogated in renal cancer; however, these studies focused primarily on the role of BCL-2 because BCL-2 inhibition had been a successful strategy to target other cancers (46-48). Our findings, relying on unbiased screens and subsequent validations, demonstrated that kidney cancer cells exhibit much greater dependence on BCL-XL than BCL-2. These findings refocus attention on BCL-X blockers as potential therapeutic agents in kidney cancer. 
Our findings indicate that $\sim 30 \%$ of human renal tumors, especially those that represent the more aggressive mesenchymal signatures, are likely to be responsive to $\mathrm{Bcl}-\mathrm{xL}$ inhibition. To further this idea, we report a number of biomarkers that can be measured in tumor biopsies, including p21 expression (as a marker for p53 activity) and mesenchymal mRNA gene expression signatures. We also demonstrate the utility of the ex vivo $\mathrm{BH} 3$-profiling assay as a faithful predictor of $B C L-X\llcorner$ dependency in renal cells. Importantly, this assay has been optimized for interrogation of apoptotic dependencies in human cancer samples $(29,49)$, and can be easily adapted for human renal tumors. Together, these assays provide us numerous future opportunities, which were beyond the scope of this initial study, to develop clinically usable biomarkers to identify cohorts of $B C L-X L$ dependent renal tumors.

The clinical use of BCL- $\mathrm{X}$ blockers has been limited due to adverse events, raising concerns about the potential clinical utility of this approach. However, most of these studies were performed using earlier versions of BCL-XL blockers, which also had effects on BCL-2 and other BCL-2-family proteins including BCL-w. More specific BCL$X_{L}$ inhibitors (such as the highly potent agent A-1331852) may have reduced toxicities when they are evaluated in vivo. Thrombocytopenia will continue to be a concern given that platelets are highly dependent on $B C L-X_{L}$ for survival and quickly undergo apoptosis when this protein is inhibited (50). However, novel approaches that degrade $B C L-X_{L}$ in nucleated cells but not platelets reduce thrombocytopenia in animal models and are now entering clinical trials (51). These pharmacological advances, combined with assays that exploit our biological predictors of $B C L-X L$ dependence and assign 
therapy more rationally in patients most likely to respond, could enable efficacious clinical use of BCL-XL inhibitors in kidney cancer.

Why might mesenchymal cancer cells be dependent on $B C L-X_{L}$ ? In normal tissues, cellular shedding in response to disruption of epithelial organization triggers cell death pathways in dislodged cells. This process is called Anoikis (greek for "homelessness") [fig. S13 (left)]. Cancer cells undergoing epithelial-mesenchymal transition, should likewise be primed for cell death. We speculate that mesenchymal kidney cancer cells possibly begin to resemble an anoikis-like state and select for BCLX's anti-apoptotic activity to ensure their survival [fig. S13 (right)]. Altogether, our observations justify testing the use of $B C L-X_{L}$ blockers in the management of the mesenchymal - more aggressive - kidney tumors.

\section{Acknowledgments:}

We thank Dr. Ruth Keri (Cleveland Clinic) for the BCL-XL expression construct and Dr. Alexandru Almasan (Cleveland Clinic) for the SU-DHL-6 cell line. We thank Dr. Christopher Weight (Cleveland Clinic) for critiques on the clinical relevance of these findings. Funding: AAC is supported by seed money from the Cleveland Clinic Foundation, the Case Comprehensive Cancer Center Jump Start Award (RES515351), the DoD's Kidney Cancer Research Program Early Career Investigator award (W81XWH-20-1-0804), the Velosano pilot award, and the $\mathrm{V}$ foundation scholar award (V2020-011). W.G.K. is supported by an NIH R35CA210068 and NIH P50CA101942 and is an HHMI Investigator. K.A.S. is supported by Harvard T.H. Chan School of Public Health Dean's Fund for Scientific Advancement, Andrew McDonough B+ Foundation, 
Making Headway Foundation St. Baldrick's Research Grant, NIH/NCl R00CA188679, and NIH/NIDDK R01DK125263. Author contributions: SM and LS designed and performed cell-based experiments. TR modeled the drug-drug interactions. CF and KAS performed $\mathrm{BH} 3$ profiling studies. JMKB and FV analyzed the Achilles data to generate the candidate gene list. TG, AAC, and WGK designed experiments, analyzed results, and wrote the manuscript. Competing interests: The authors declare no conflicts of interest with the data presented in this study. Data and materials availability: All gene expression data are deposited into the Gene Expression Omnibus (GSE173618). 


\section{References}

1. R. L. Siegel, K. D. Miller, H. E. Fuchs, A. Jemal, Cancer statistics, 2022. CA Cancer J Clin 72, 7-33 (2022).

2. T. K. Choueiri et al., Nivolumab plus Cabozantinib versus Sunitinib for Advanced Renal-Cell Carcinoma. N Engl J Med 384, 829-841 (2021).

3. R. Motzer et al., Lenvatinib plus Pembrolizumab or Everolimus for Advanced Renal Cell Carcinoma. N Engl J Med 384, 1289-1300 (2021).

4. B. I. Rini et al., Pembrolizumab plus Axitinib versus Sunitinib for Advanced Renal-Cell Carcinoma. N Engl J Med 380, 1116-1127 (2019).

5. W. M. Linehan, C. J. Ricketts, The Cancer Genome Atlas of renal cell carcinoma: findings and clinical implications. Nat Rev Urol 16, 539-552 (2019).

6. A. A. Chakraborty et al., HIF activation causes synthetic lethality between the VHL tumor suppressor and the EZH1 histone methyltransferase. Sci Transl Med 9, (2017).

7. D. A. Chan et al., Targeting GLUT1 and the Warburg effect in renal cell carcinoma by chemical synthetic lethality. Sci Transl Med 3, 94ra70 (2011).

8. S. Turcotte et al., A molecule targeting VHL-deficient renal cell carcinoma that induces autophagy. Cancer Cell 14, 90-102 (2008).

9. C. J. Schofield, P. J. Ratcliffe, Oxygen sensing by HIF hydroxylases. Nature reviews. Molecular cell biology 5, 343-354 (2004).

10. W. G. Kaelin, Jr., P. J. Ratcliffe, Oxygen sensing by metazoans: the central role of the HIF hydroxylase pathway. Molecular cell 30, 393-402 (2008).

11. K. Kondo, W. Y. Kim, M. Lechpammer, W. G. Kaelin, Jr., Inhibition of HIF2alpha is sufficient to suppress pVHL-defective tumor growth. PLoS biology 1, E83 (2003).

12. J. D. Gordan, J. A. Bertout, C. J. Hu, J. A. Diehl, M. C. Simon, HIF-2alpha promotes hypoxic cell proliferation by enhancing c-myc transcriptional activity. Cancer Cell 11, 335-347 (2007).

13. T. H. Scheuermann et al., Allosteric inhibition of hypoxia inducible factor-2 with small molecules. Nature chemical biology 9, 271-276 (2013).

14. T. H. Scheuermann et al., Isoform-Selective and Stereoselective Inhibition of Hypoxia Inducible Factor-2. J Med Chem 58, 5930-5941 (2015).

15. W. Chen et al., Targeting renal cell carcinoma with a HIF-2 antagonist. Nature 539, 112-117 (2016).

16. H. Cho et al., On-target efficacy of a HIF-2alpha antagonist in preclinical kidney cancer models. Nature 539, 107-111 (2016).

17. K. D. Courtney et al., Phase I Dose-Escalation Trial of PT2385, a First-in-Class Hypoxia-Inducible Factor-2alpha Antagonist in Patients With Previously Treated Advanced Clear Cell Renal Cell Carcinoma. J Clin Oncol, JCO2017742627 (2017).

18. W. G. Kaelin, Jr., HIF2 Inhibitor Joins the Kidney Cancer Armamentarium. J Clin Oncol 36, 908-910 (2018).

19. G. S. Cowley et al., Parallel genome-scale loss of function screens in 216 cancer cell lines for the identification of context-specific genetic dependencies. Sci Data 1, 140035 (2014). 
20. A. Tsherniak et al., Defining a Cancer Dependency Map. Cell 170, 564-576 e516 (2017).

21. E. R. McDonald, 3rd et al., Project DRIVE: A Compendium of Cancer Dependencies and Synthetic Lethal Relationships Uncovered by Large-Scale, Deep RNAi Screening. Cell 170, 577-592 e510 (2017).

22. W. G. Kaelin, Jr., Molecular biology. Use and abuse of RNAi to study mammalian gene function. Science 337, 421-422 (2012).

23. M. Paces-Fessy, M. Fabre, C. Lesaulnier, S. Cereghini, Hnf1b and Pax2 cooperate to control different pathways in kidney and ureter morphogenesis. Human molecular genetics 21, 3143-3155 (2012).

24. O. Wessely, U. Tran, Xenopus pronephros development--past, present, and future. Pediatr Nephrol 26, 1545-1551 (2011).

25. T. Oltersdorf et al., An inhibitor of Bcl-2 family proteins induces regression of solid tumours. Nature 435, 677-681 (2005).

26. C. Tse et al., ABT-263: a potent and orally bioavailable Bcl-2 family inhibitor. Cancer Res 68, 3421-3428 (2008).

27. A. J. Souers et al., ABT-199, a potent and selective BCL-2 inhibitor, achieves antitumor activity while sparing platelets. Nature medicine 19, 202-208 (2013).

28. L. Wang et al., Discovery of A-1331852, a First-in-Class, Potent, and OrallyBioavailable BCL-XL Inhibitor. ACS Med Chem Lett 11, 1829-1836 (2020).

29. T. Ni Chonghaile et al., Pretreatment mitochondrial priming correlates with clinical response to cytotoxic chemotherapy. Science 334, 1129-1133 (2011).

30. R. J. Motzer, P. Russo, Systemic therapy for renal cell carcinoma. J Urol 163, 408-417 (2000).

31. C. I. J. A. o. a. b. Bliss, The toxicity of poisons applied jointly 1. 26, 585-615 (1939).

32. S. J. F. t. a. o. Loewe, The problem of synergism and antagonism of combined drugs Arzneimittelforschung 3: 285-290. (1953).

33. M. C. Berenbaum, What is synergy? Pharmacol Rev 41, 93-141 (1989).

34. B. Yadav, K. Wennerberg, T. Aittokallio, J. Tang, Searching for Drug Synergy in Complex Dose-Response Landscapes Using an Interaction Potency Model. Comput Struct Biotechnol J 13, 504-513 (2015).

35. A. lanevski, L. He, T. Aittokallio, J. Tang, SynergyFinder: a web application for analyzing drug combination dose-response matrix data. Bioinformatics 33, 24132415 (2017).

36. A. Liberzon, A description of the Molecular Signatures Database (MSigDB) Web site. Methods in molecular biology 1150, 153-160 (2014).

37. A. Subramanian et al., Gene set enrichment analysis: a knowledge-based approach for interpreting genome-wide expression profiles. Proc Natl Acad Sci U $S$ A 102, 15545-15550 (2005).

38. K. A. Sarosiek et al., Developmental Regulation of Mitochondrial Apoptosis by cMyc Governs Age- and Tissue-Specific Sensitivity to Cancer Therapeutics. Cancer Cell 31, 142-156 (2017).

39. F. J. Sanchez-Rivera et al., Mitochondrial apoptotic priming is a key determinant of cell fate upon p53 restoration. Proc Natl Acad Sci U S A 118, (2021). 
40. J. Le Pen et al., Constitutive p53 heightens mitochondrial apoptotic priming and favors cell death induction by $\mathrm{BH} 3$ mimetic inhibitors of $\mathrm{BCL}-\mathrm{xL}$. Cell death \& disease 7, e2083 (2016).

41. N. Chen et al., BCL-xL is a target gene regulated by hypoxia-inducible factor1 alpha\}. The Journal of biological chemistry 284, 10004-10012 (2009).

42. K. Guo et al., Hypoxia induces the expression of the pro-apoptotic gene BNIP3. Cell Death Differ 8, 367-376 (2001).

43. M. A. Esteban et al., Regulation of E-cadherin expression by VHL and hypoxiainducible factor. Cancer Res 66, 3567-3575 (2006).

44. S. Schokrpur et al., CRISPR-Mediated VHL Knockout Generates an Improved Model for Metastatic Renal Cell Carcinoma. Sci Rep 6, 29032 (2016).

45. J. D. Leverson et al., Exploiting selective BCL-2 family inhibitors to dissect cell survival dependencies and define improved strategies for cancer therapy. Sci Transl Med 7, 279 ra240 (2015).

46. T. Itoi, K. Yamana, V. Bilim, K. Takahashi, F. Tomita, Impact of frequent Bcl-2 expression on better prognosis in renal cell carcinoma patients. $\mathrm{Br} J$ Cancer $\mathbf{9 0}$, 200-205 (2004).

47. I. Kausch et al., Inhibition of bcl-2 enhances the efficacy of chemotherapy in renal cell carcinoma. Eur Urol 47, 703-709 (2005).

48. A. H. Nayman et al., Dual-Inhibition of mTOR and Bcl-2 Enhances the Anti-tumor Effect of Everolimus against Renal Cell Carcinoma In Vitro and In Vivo. J Cancer 10, 1466-1478 (2019).

49. C. Fraser, J. Ryan, K. Sarosiek, BH3 Profiling: A Functional Assay to Measure Apoptotic Priming and Dependencies. Methods in molecular biology 1877, 61-76 (2019).

50. K. A. Sarosiek, A. Letai, Directly targeting the mitochondrial pathway of apoptosis for cancer therapy using $\mathrm{BH} 3$ mimetics - recent successes, current challenges and future promise. The FEBS journal 283, 3523-3533 (2016).

51. S. Khan et al., A selective BCL-XL PROTAC degrader achieves safe and potent antitumor activity. Nature medicine 25, 1938-1947 (2019). 


\section{MAIN FIGURE LEGENDS}

FIGURE 1. BCL-XL is a Strong Dependency in a Subset of Kidney-lineage Cancer

Cells. (A and B) List of dependencies enriched for selectivity in a subset of kidney lineage cells, as indicated by Likelihood Ratio Test (LRT) scores > 100 (A) and comparison of $B C L-X_{L}$ dependency in the RNAi versus CRISPR/Cas9 dependency maps, in the indicated kidney cancer cell lines (B). Negative scores indicate a dependency with scores $<-1$ (strong, green oval), -0.5 to -1.0 (intermediate, blue oval), and >-0.5 (neutral/resistant, red triangle), annotated based on the CRISPR/Cas9 dataset. (C and D) Immunoblots (C) and crystal violet staining (D) of the indicated cell lines that were lentivirally transduced to express shRNAs targeting the indicated BCL-2-family genes or non-targeting controls (Con). ( $\mathbf{E}$ to $\mathbf{G})$ Immunoblots $(\mathbf{E})$ and crystal violet staining ( $\mathbf{F}$ and G) of the indicated cell lines that were lentivirally transduced to express the indicated shRNA-resistant versions of BCL-XL or GFP, as a control, followed by lentiviral expression of the indicated shRNAs. (C) and (E) were done 3 days post infection with the shRNAexpressing lentiviral particles. (D), (F), and (G) were done 3 days post selection for A498, CAKI-2, and UMRC-6 cells, and 7-10 days post selection for UMRC-2 cells.

FIGURE 2. BCL-XL Inhibition Promotes Apoptotic Cell Death in a Subset of ccRCCs, (A and B) Percent cell death, relative to the DMSO-treated control cells, determined using the XTT assay in the indicated ccRCC cell lines that were treated with the indicated concentrations of the $B C L-X L$ inhibitor A-1331852 (A) or the BCL-2 inhibitor ABT-199 (B) for 3 days. (C and D) JC1 fluorescence measurement at the indicated time-points in CAKI2 (C) and UMRC-2 cells (D) that were exposed to Alamethicin (Alam) (positive control), 
DMSO (negative control), sensitizer $\mathrm{BH} 3$ peptides (MS1 targeting MCL-1 and Hrk targeting $\mathrm{BCL}-\mathrm{XL}_{\mathrm{L}}$ ), or the small molecule BCL-XL inhibitor Wehi539.

FIGURE 3. BCL-XL Inhibition Chemosensitizes ccRCCs to Chemotherapeutics. (A, C, E, and G) Mean synergism score calculated across four independent models (ZIP, HSA, Loewe, Bliss) for 5-fluorouracil (5-FU) (A and $\mathbf{C}$ ) and Docetaxel (E and $\mathbf{G})$ at the indicated doses in combination with A-1331852. (B, D, F, and H) Percent cell viability and calculated dose-response curves in cells cultured in the presence of the indicated concentrations of the two molecules for 72 hours. Results for UMRC-2 (A, B, E, and F) and OSRC2 (C, D, G, and $\mathbf{H})$ were calculated from $\geq 3$ biological replicates. Error bars represent mean $\pm S D$.

FIGURE 4. Transcriptomics Analysis Identifies Correlates Associated with BCL-XL

Dependency. (A) Schema (top) and number of genes that were identified as differentially expressed (bottom, $\geq 1.5$-fold and FDR $\leq 10 \%$, as determined by RNA-Seq) in the indicated BCL-XL inhibitor 'Sensitive' (S) versus 'Insensitive' (I), under untreated basal conditions or upon acute treatment with A-1331852 (as described in the Methods). (B and C) Lollipop plot (B) depicting statistically significant results (NES $\geq 1.5$ and FDR $\leq 10 \%$ ) of Gene Set Enrichment Analysis comparing RNA-Seq data from S vs I cells that were either untreated (open circles) or A-1331852 treated (filled circles) and representative gene sets that were enriched in the Sensitive cells (top, red) (C) or the Insensitive cells (bottom, blue) (D). 
FIGURE 5. A Mesenchymal Cell State Promotes BCL-XL Dependency. (A) Schema showing experimental design to address necessity of mesenchymal state in promoting BCL-XL dependency. (B) CD44 levels, as determined by flow cytometry, in the indicated cCRCC cells that were treated with $1 \mu \mathrm{M}$ ATRA for 3 days. (C) Cell viability, relative to untreated DMSO controls, in the indicated cells treated with A-1331852 for 7 days. (D) Schema showing experimental design to address sufficiency of mesenchymal state in promoting Bcl-xL dependency. (E) CD44 levels, as determined by flow cytometry, in the indicated ccRCC cells that were treated with $10 \mathrm{ng} / \mathrm{ml}$ TGF $\beta$ for 3 days. (F) Cell viability, relative to untreated DMSO controls, in the indicated cells treated with A-1331852 for 7 days. Data in (B) and (E) was normalized to CD44 levels in the untreated (Vehicle) control and was compared using the Student's t-test ( $n \geq 3$, bar represents Mean, $p$-values are indicated). (C) and (F) were normalized for batch effects and compared using linear regression ( $n \geq 3$, dotted line represents best fit, $p$-values indicated are for difference in slopes i.e., interaction between A-1331852 and ATRA or TGF $\beta$ )

FIGURE 6. BCL-XL Dependency Signatures are Evident in ccRCC Clinical Specimens. (A) Schema indicating the computational overlay of differentially-expressed genes, as determined in 4A, onto renal cancer gene expression data mined from TCGA. (B) Principle-component analysis showing segregation of tumors based on their subtype (left), and then within ccRCC (C) by similarity in gene signature to Sensitive (red) versus insensitive (blue) lines (right). (D and E) Median gene expression levels of the indicated gene sets (D) and Kaplan-Meier curves depicting clinical outcomes (E) in patients with tumors resembling the BCL- $X_{L}$ inhibitor Sensitive versus Insensitive cell lines. (A) and (D) 
were compared using the Student's t-test. In $(\mathbf{E})$ Sensitive $(\mathrm{n}=171)$ and Insensitive $(\mathrm{n}=$ 348) patients were compared by the log-rank test.

Figure 7. Pharmacological BCL-XL Inhibition Impedes Tumor Growth. Tumor volumes at Day 0 , start of dosing $(\mathbf{A})$, change in tumor volume at the indicated time points after A-1331852 dosing at $25 \mathrm{mg} / \mathrm{kg}$, twice a day, by oral gavage (B), time for tumor volume to triple on the indicated dosing regimens $(\mathbf{C})$, and photomicrographs of harvested tumors and the indicated animal organs (D), in NOD/SCID mice that were inoculated subcutaneously with pVHL-deficient UMRC-2 cells. (E and F) Histological analysis by H\&E staining and immunohistochemistry, showing DAPI (blue) and Cleaved Caspase 3 (green) staining (bottom panel), of tumors harvested from animals dosed with Vehicle control (E) or A-1331852 (F), as indicated. 
bioRxiv preprint doi: https://doi.org/10.1101/2022.01.29.478337; this version posted January 31, 2022. The copyright holder for this preprint (which was not certified by peer review) is the author/funder. All rights reserved. No reuse allowed without permission.

\section{FIGURE 1}

A

\begin{tabular}{|c|c|c|c|}
\hline entrez_id & symbol & Gene Description & |RNAi_LRT Druggable \\
\hline 6928 & HNF1B & HNF1 homeobox B & $\begin{array}{ll}537.5 \mathrm{NO} \\
\end{array}$ \\
\hline 7849 & PAX8 & paired box 8 & $308.6 \mathrm{NO}$ \\
\hline 6209 & RPS15 & ribosomal protein S15 & $198.2 \mathrm{NO}$ \\
\hline 9045 & RPL14 & ribosomal protein L14 & $190.0 \mathrm{NO}$ \\
\hline 8662 & EIF3B & eukaryotic translation initiation factor 3 subunit $B$ & $\begin{array}{ll}172.7 & \mathrm{NO} \\
\end{array}$ \\
\hline 598 & BCL2L1 & BCL2 like 1 & 172.0 YES \\
\hline 9349 & RPL23 & ribosomal protein $\mathrm{L} 23$ & $160.9 \mathrm{NO}$ \\
\hline 6223 & RPS19 & ribosomal protein $\mathrm{S} 19$ & $158.5 \mathrm{NO}$ \\
\hline 6633 & SNRPD2 & small nuclear ribonucleoprotein D2 polypeptide & \begin{tabular}{l|l}
156.2 & $\mathrm{NO}$
\end{tabular} \\
\hline 3685 & ITGAV & integrin subunit alpha $\mathrm{V}$ & 145.3 YES \\
\hline
\end{tabular}

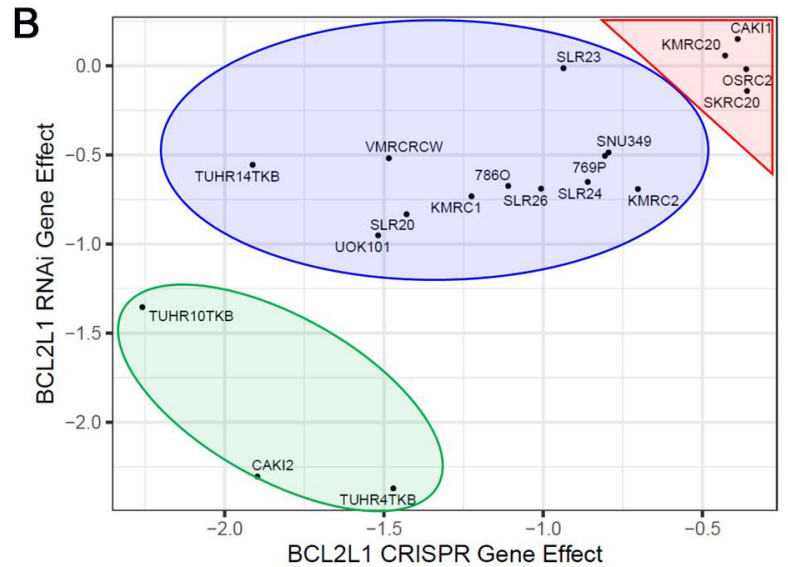

C

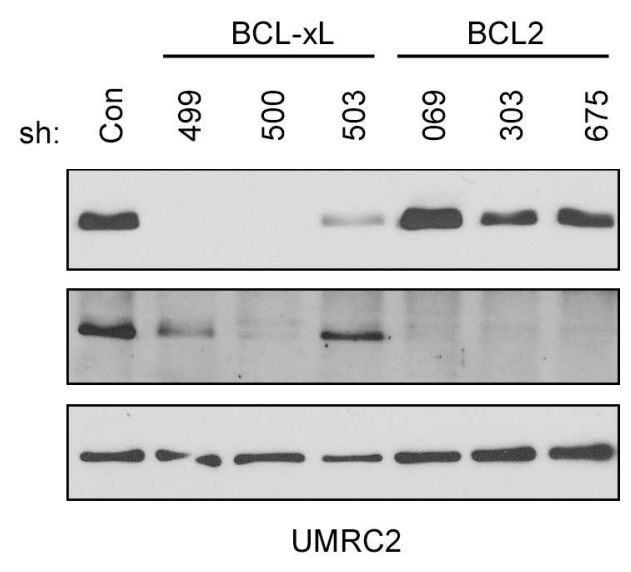

BCL-xL

BCL2

Vinculin

D

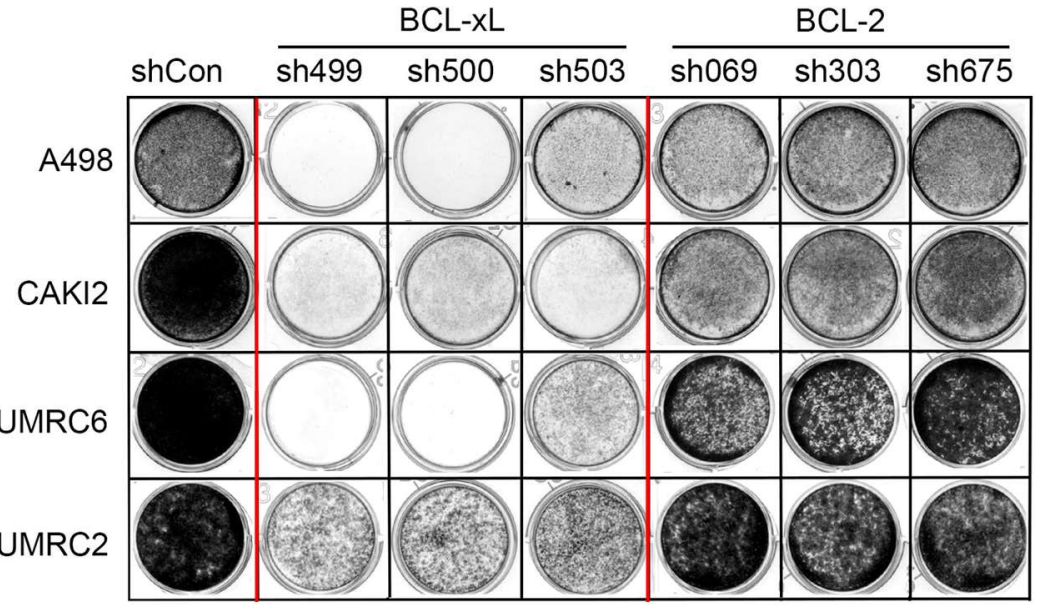

F

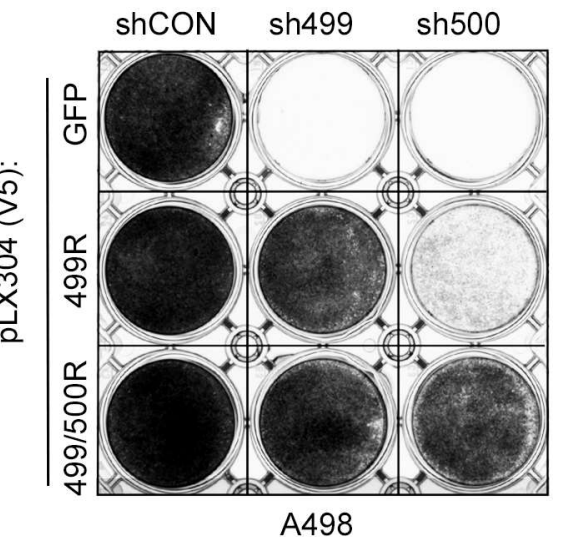

G

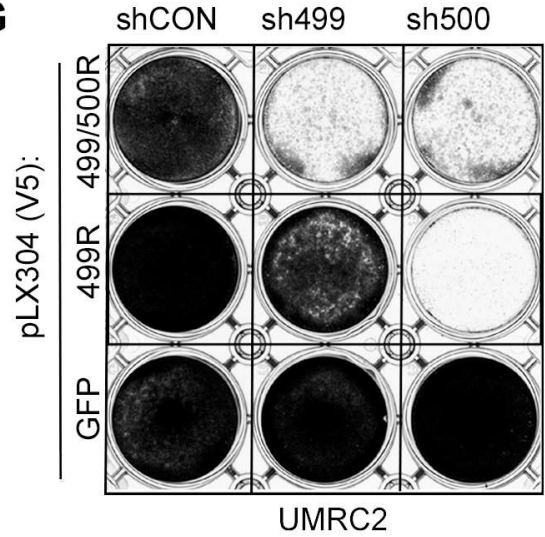




\section{FIGURE 2}

A

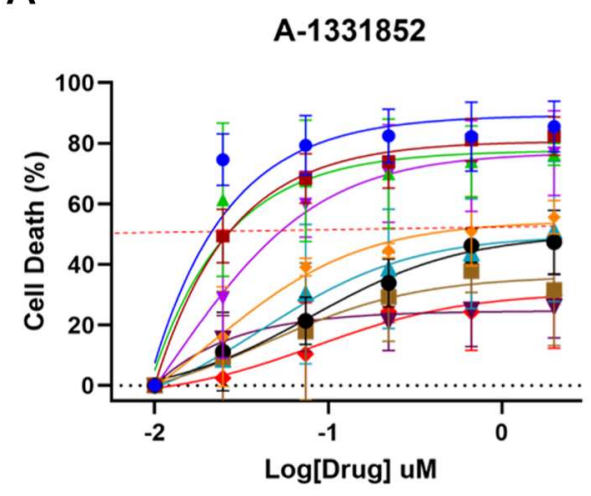

C

CAKI2

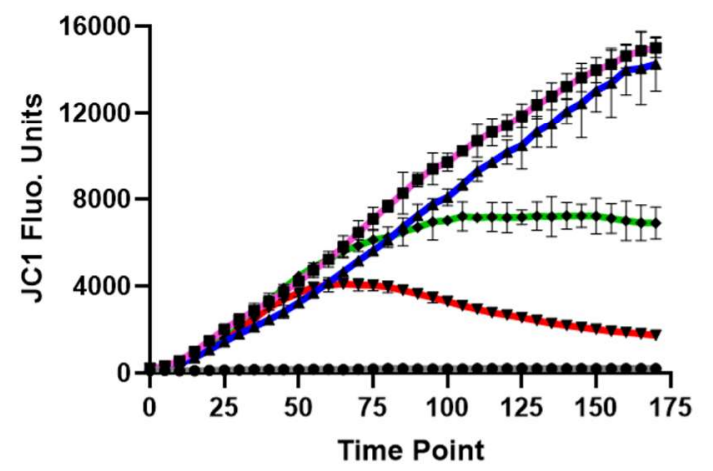
$\rightarrow 7860$

- UMRC6

- UMRC2

- UOK101

$\rightarrow$ OSRC2

- RCC4
B Cell Line BCL-xL inhibition - CAKI2 Sensitive - A498 Sensitive ^ TUHR4 Sensitive $\rightarrow$ SLR23 Sensitive Intermediate Intermediate Insensitive Insensitive Insensitive Insensitive

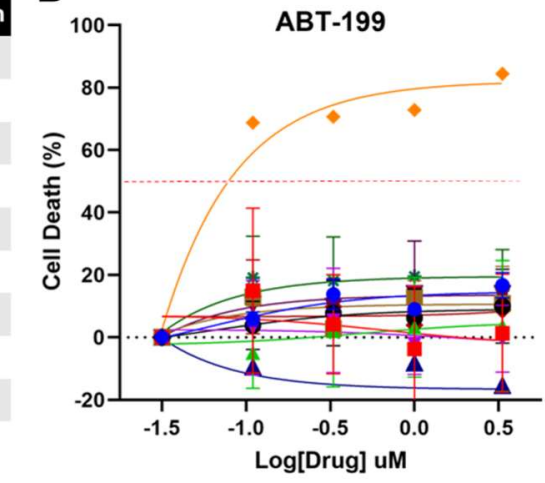

Cell Line BCL-2 inhibition

D

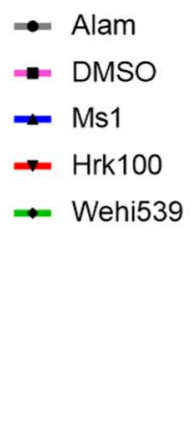

\section{UMRC2}

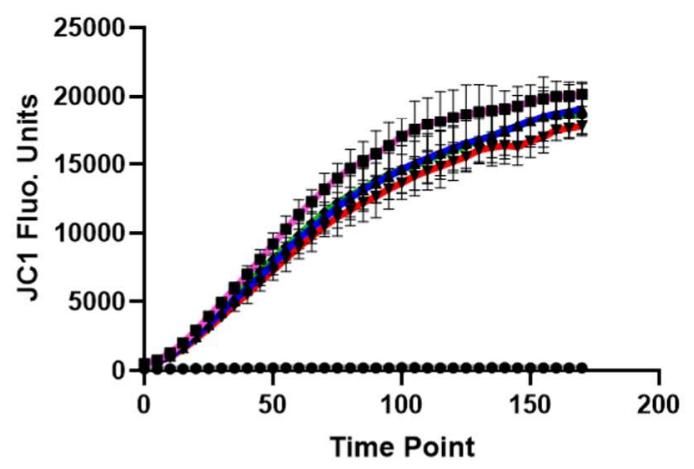

- Alam
- DMSO
$=$ Ms1
- Hrk100
- Wehi539 
bioRxiv preprint doi: https://doi.org/10.1101/2022.01.29.478337; this version posted January 31, 2022. The copyright holder for this preprint (which was not certified by peer review) is the author/funder. All rights reserved. No reuse allowed without permission.

\section{FIGURE 3}

A

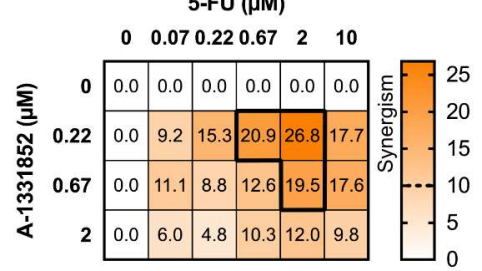

E

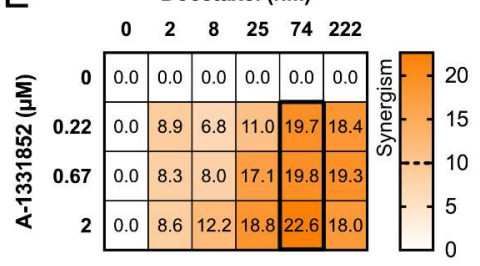

B

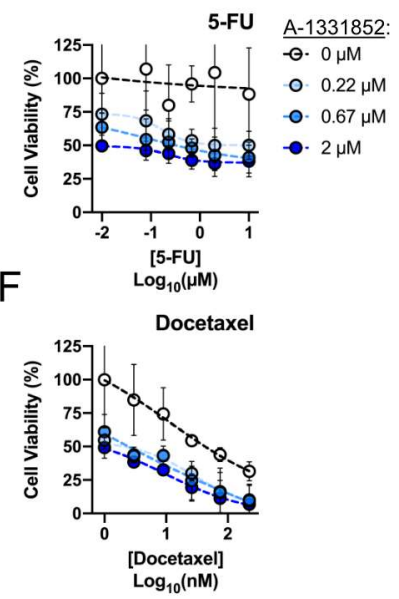

C

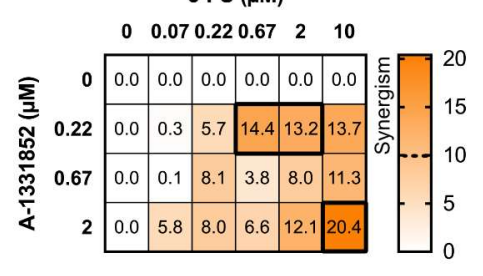

G

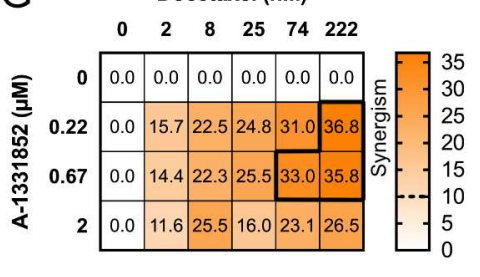

D
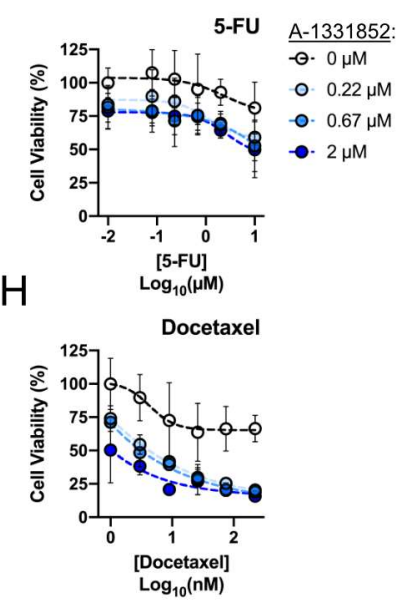
bioRxiv preprint doi: https://doi.org/10.1101/2022.01.29.478337; this version posted January 31, 2022. The copyright holder for this preprint (which was not certified by peer review) is the author/funder. All rights reserved. No reuse allowed without permission.

\section{FIGURE 4}

A

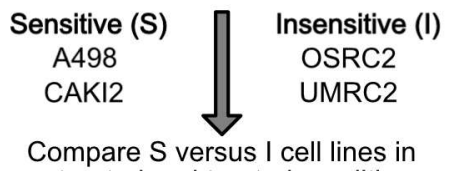

untreated and treated conditions

Untreated: S versus I

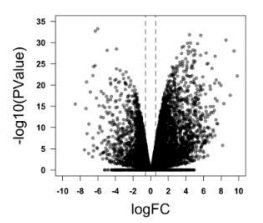

A1331852: S versus I

C
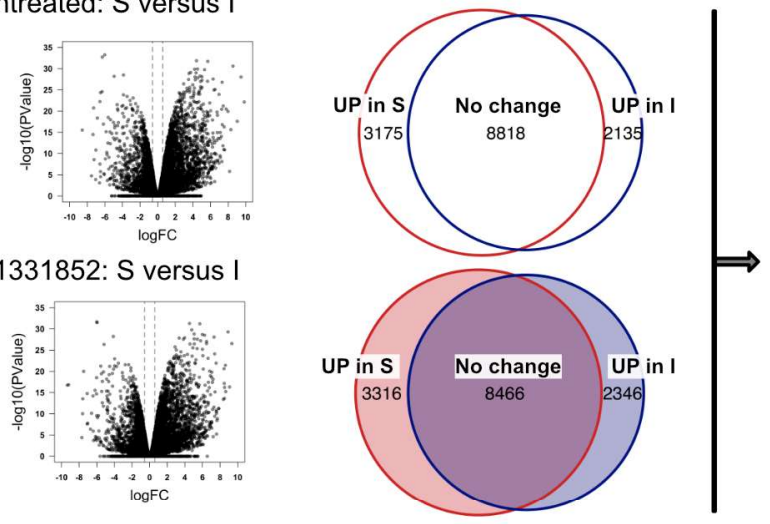

Hallmarks enriched in S untreated

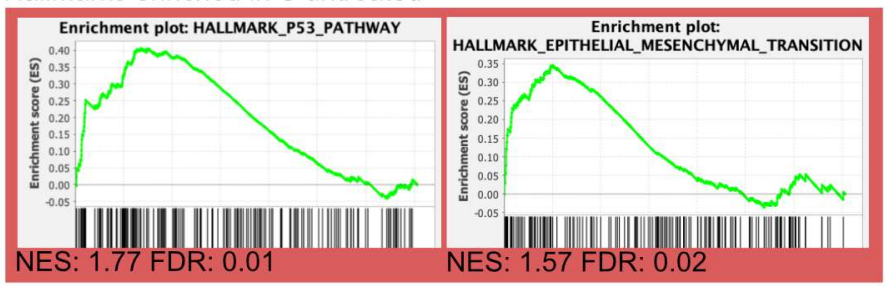

D

Hallmarks enriched in I untreated

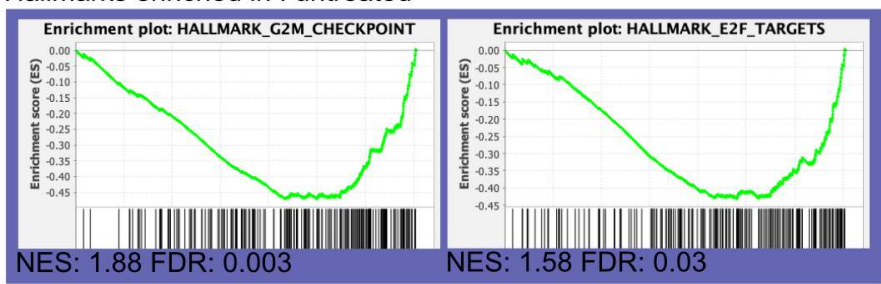

B

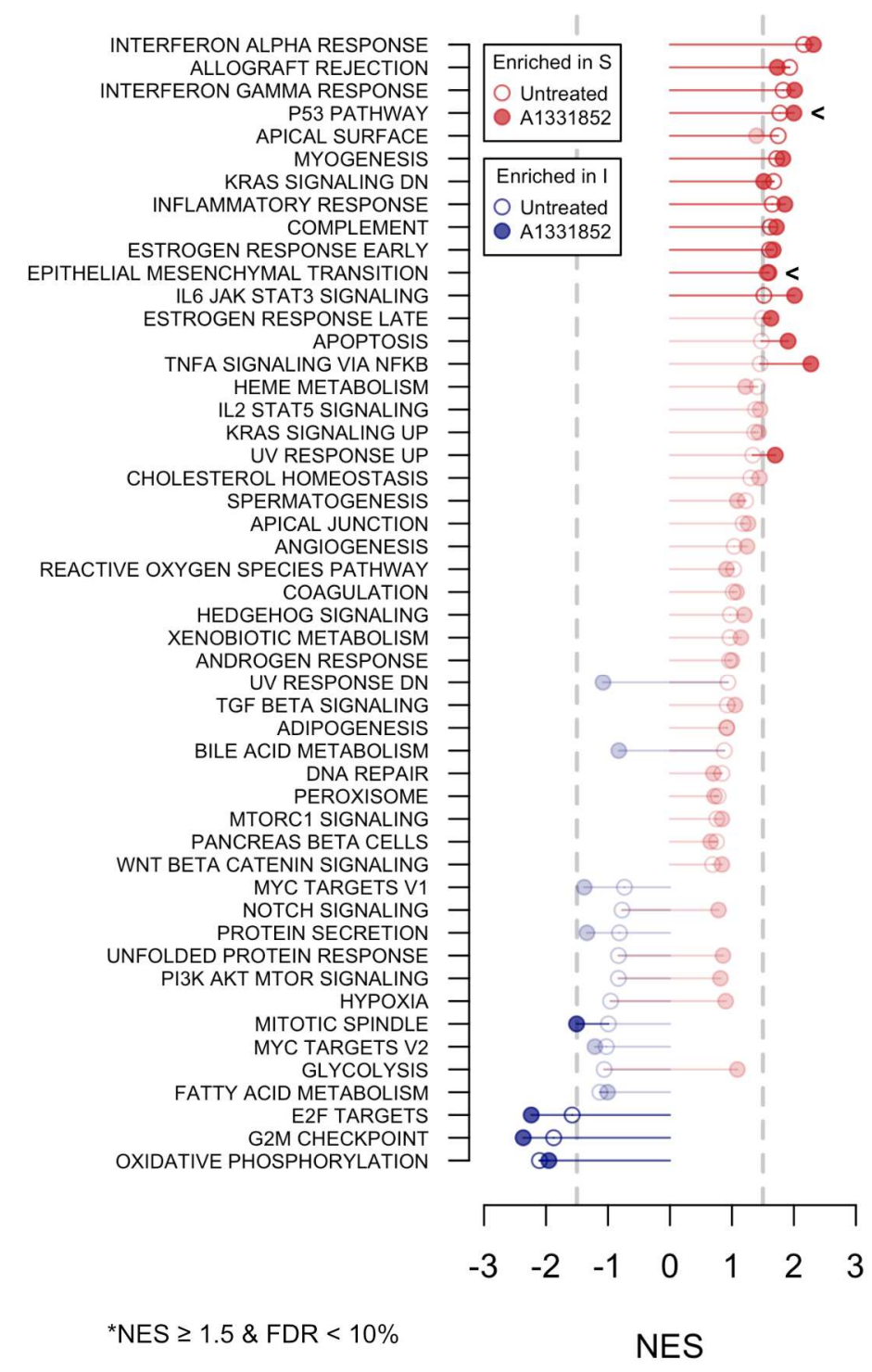




\section{FIGURE 5}

A

\section{Sensitive}

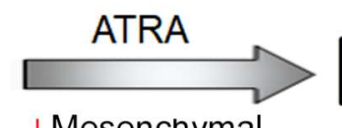

B

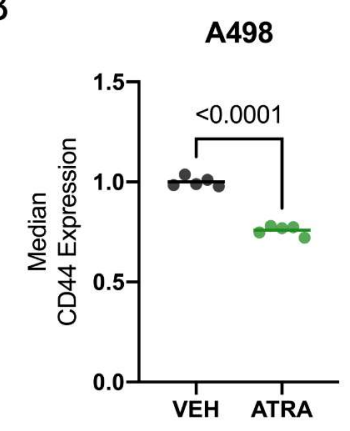

C

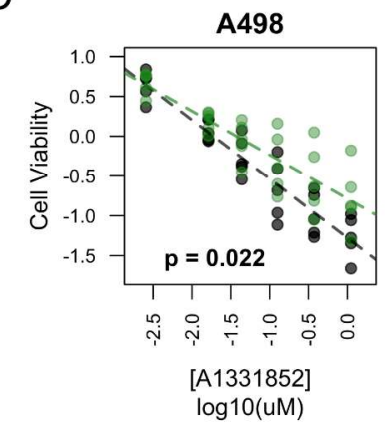

Insensitive

CAKI2
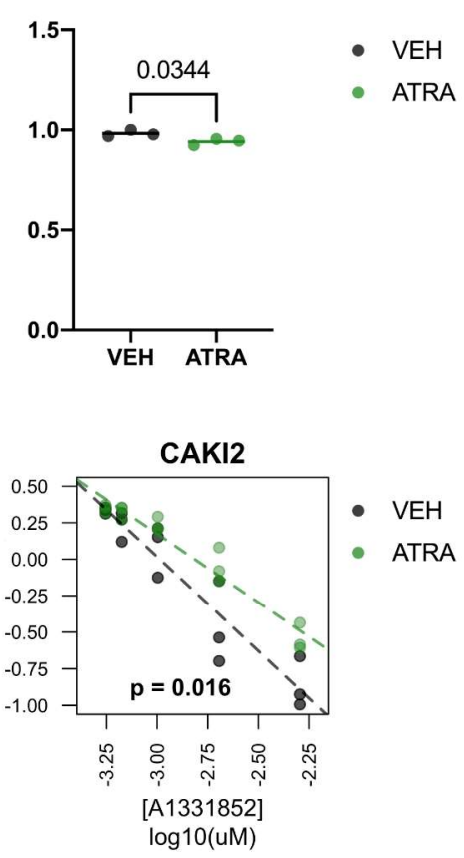

- ATRA

D

\section{Insensitive}

$E$

- VEH

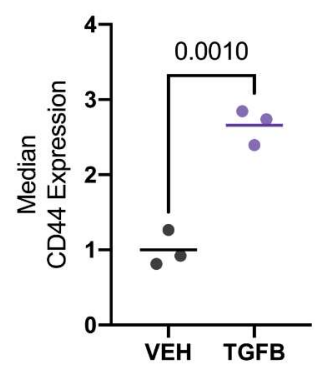

F

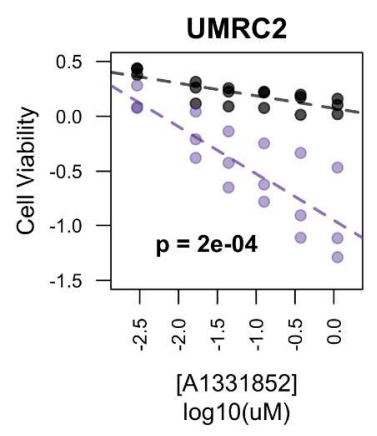

\section{Sensitive}

$\uparrow$ Mesenchymal

UMRC6
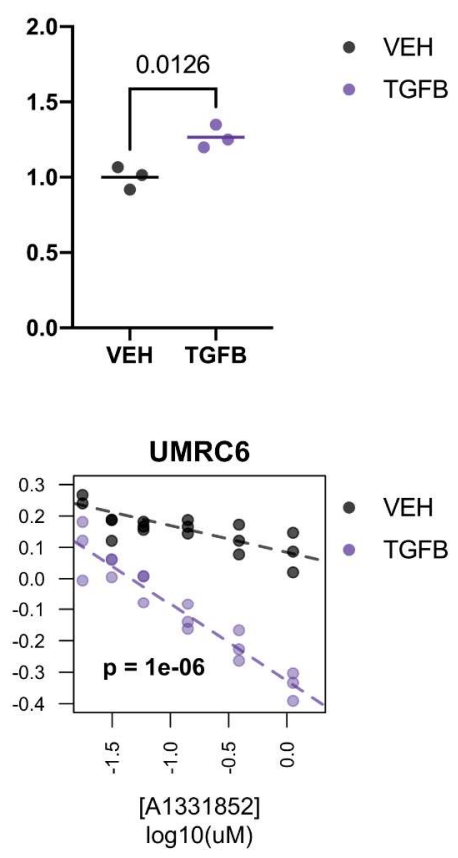
bioRxiv preprint doi: https://doi.org/10.1101/2022.01.29.478337; this version posted January 31, 2022. The copyright holder for this preprint (which was not certified by peer review) is the author/funder. All rights reserved. No reuse allowed without permission.

\section{FIGURE 6}

A

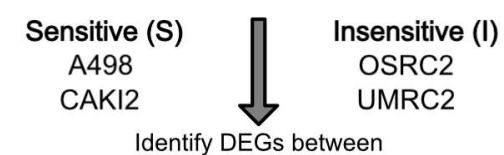
untreated $S$ versus I cell lines

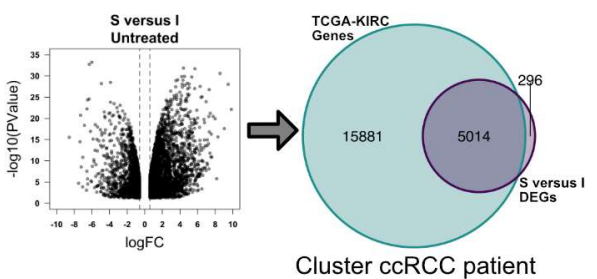

${ }^{*} \mathrm{FC} \geq 1.5 \& \mathrm{FDR}<10 \%$

D

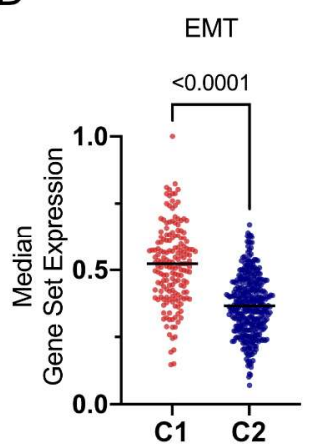

samples using DEGs

\begin{abstract}
P53 Pathway
\end{abstract}
FA Metabolism

OXPHOS
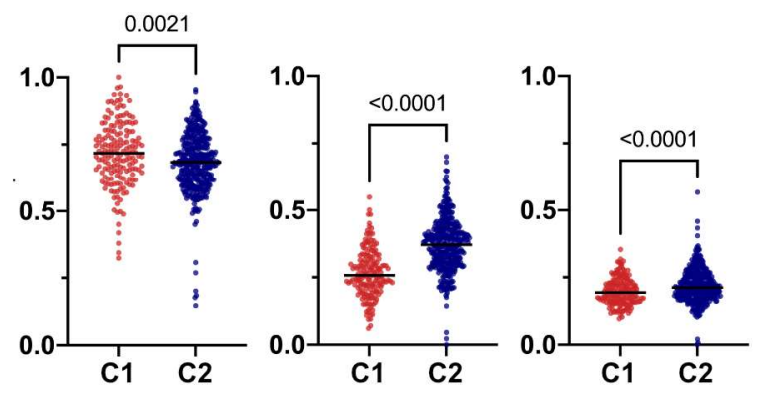

B

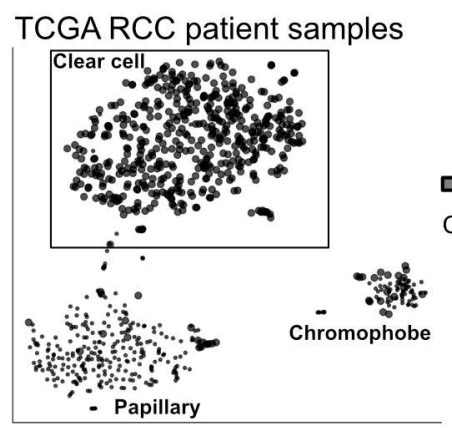

E

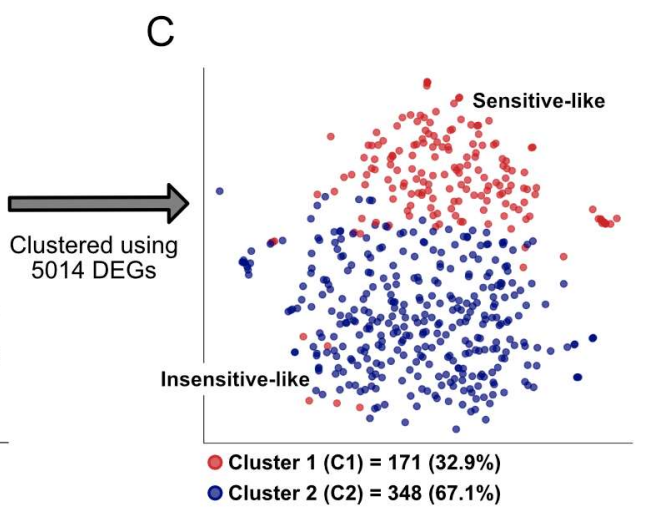




\section{FIGURE 7}

A

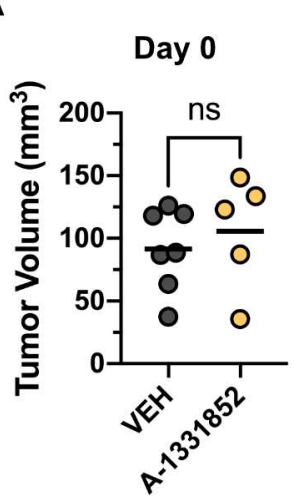

E

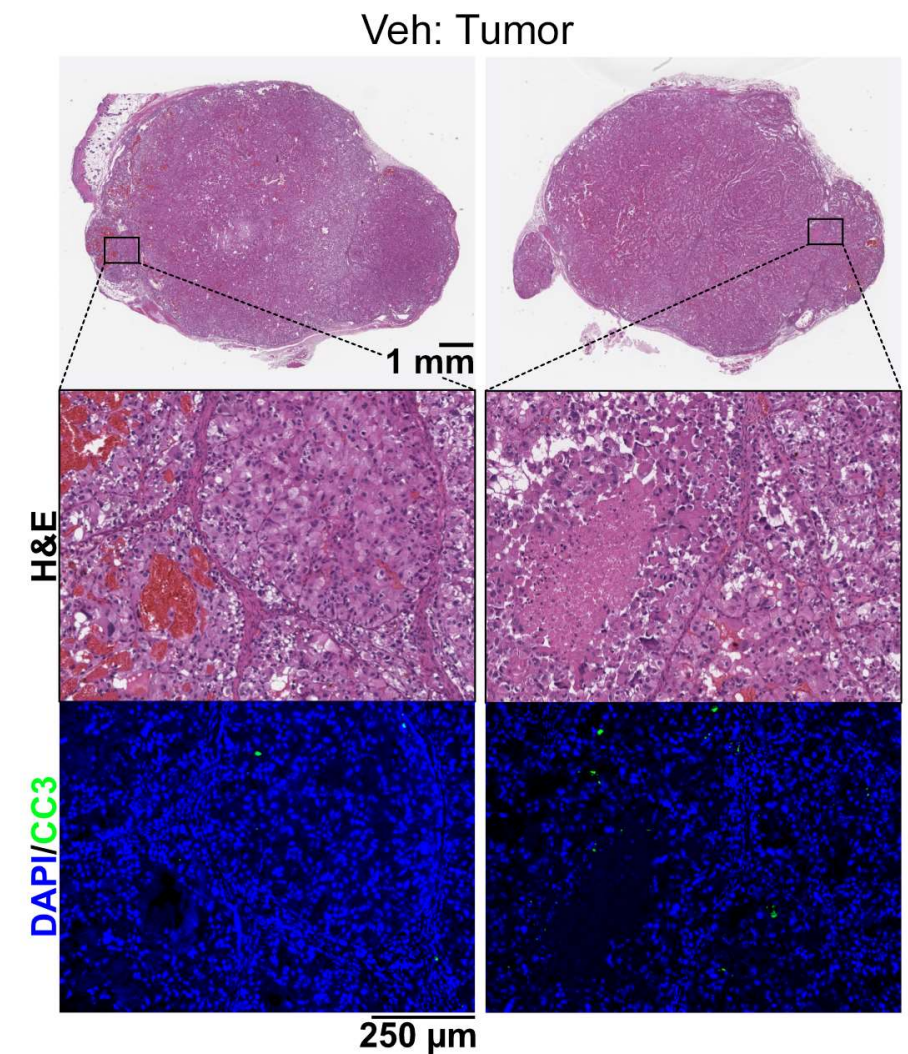

B

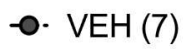

○. A-1331852 (5)

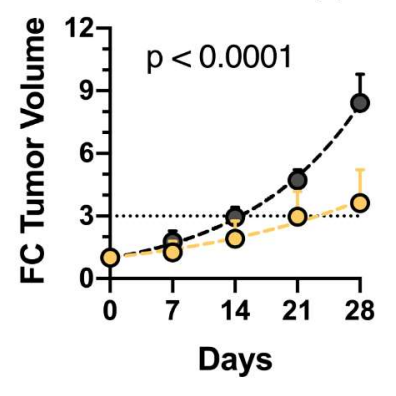

Veh: Tumor
C

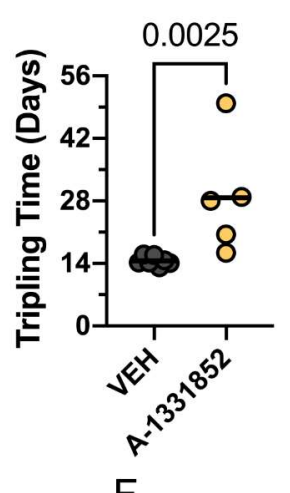

F
D

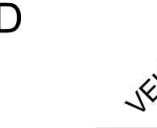<smiles>C=CC=CC=C</smiles>
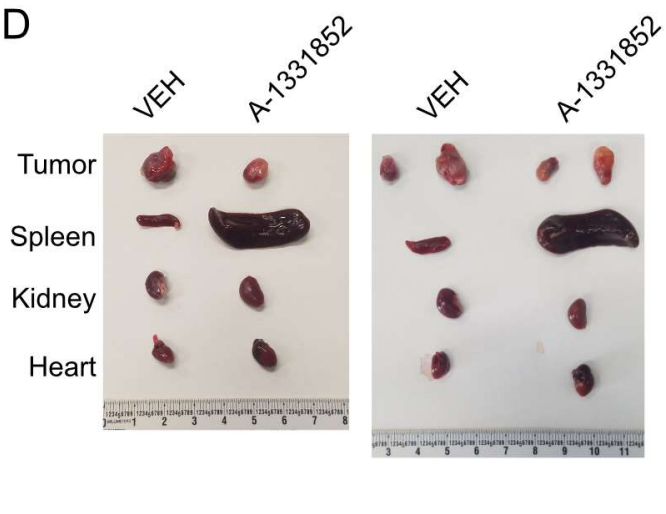

A-1331852: Tumor
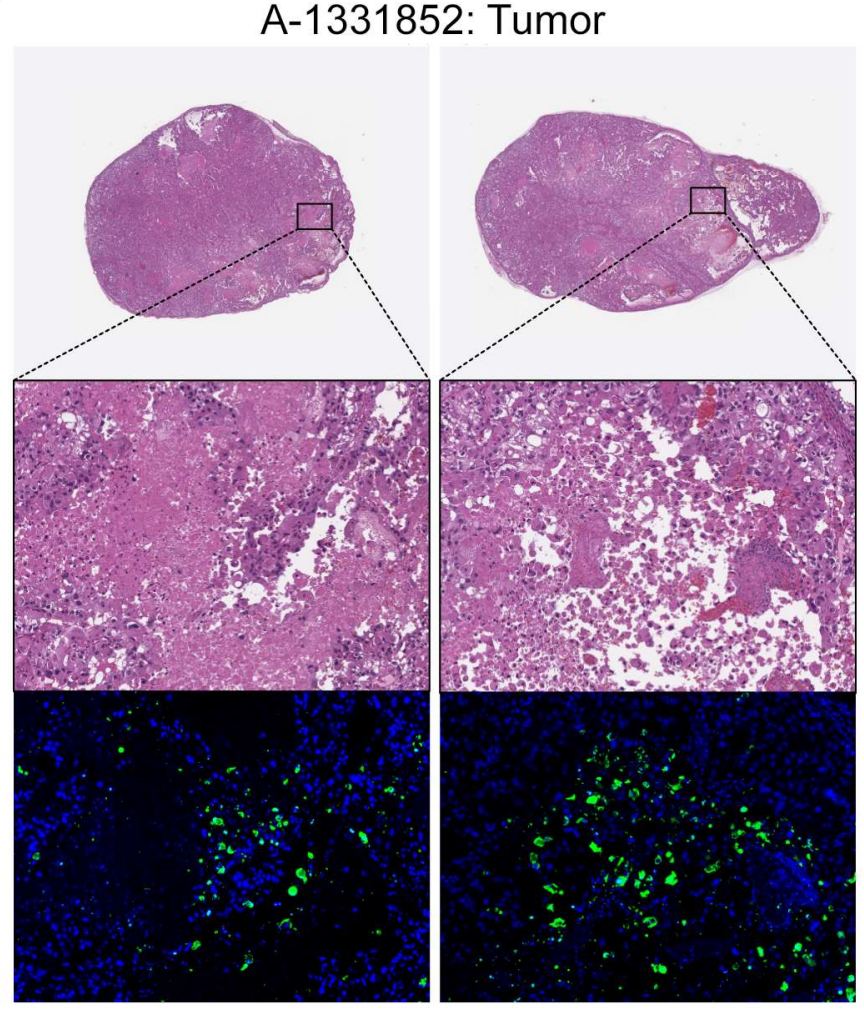